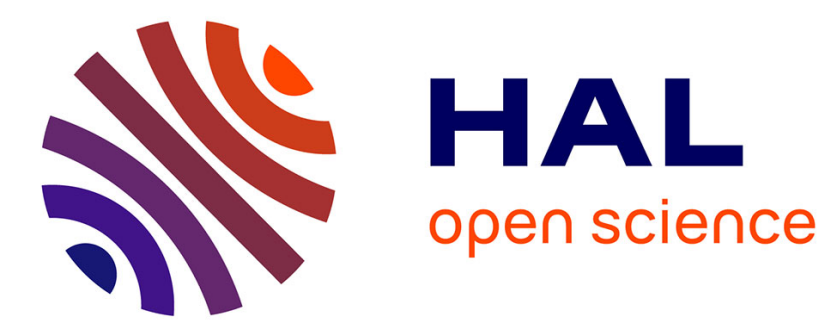

\title{
Les conflits de légitimité sous-jacents aux conflits d'usage
}

Patrice Garin, A. Richard-Ferroudji

\section{To cite this version:}

Patrice Garin, A. Richard-Ferroudji. Les conflits de légitimité sous-jacents aux conflits d'usage. La Houille Blanche - Revue internationale de l'eau, 2008, 4, p. 40 - p. $46 . \quad$ 10.1051/lhb:2008038. hal-00454470

\section{HAL Id: hal-00454470 \\ https://hal.science/hal-00454470}

Submitted on 8 Feb 2010

HAL is a multi-disciplinary open access archive for the deposit and dissemination of scientific research documents, whether they are published or not. The documents may come from teaching and research institutions in France or abroad, or from public or private research centers.
L'archive ouverte pluridisciplinaire HAL, est destinée au dépôt et à la diffusion de documents scientifiques de niveau recherche, publiés ou non, émanant des établissements d'enseignement et de recherche français ou étrangers, des laboratoires publics ou privés. 


\title{
Gestion sociale et économique de l'eau : Comment agir sur la demande
}

$10.1051 / \mathrm{lhb}: 2008038$

\section{Les conflits de légitimité sous-jacents aux conflits d'usage}

\author{
Legitimacy conflicts underlying use conflicts \\ PATRICE GARIN, AUDREY RICHARD-FERROUDJI
}

Cemagref, UMR G-EAU

361, rue Jean-François Breton, BP 5095, 34196 Montpellier Cedex

Tél. : +33 (0)4 670463 39, Fax : +33 4671664 00, e-mail : patrice.garin@cemagref.fr, audrey.richard@cemagref.fr

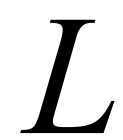

es attentes multiples de la société vis-à-vis de l'eau ont suscité de longue date des dispositifs visant à préciser ce qu'il était légitime de faire avec l'eau. Mais ces besoins et les usages évoluent, suscitant des réticences de ceux qui se sentent affectés ou lésés par ces changements. Les conflits reflètent alors une mise à l'épreuve des dispositifs anciens de régulation des usages, avec deux types de questions : ce qui était défini comme légitime et juste de faire avec l'eau l'est il toujours? Les personnes et entités impliquées dans l'élaboration et le fonctionnement de ces dispositifs sont ils légitimes à le faire?

L'approche de la justification proposée par L. Boltanski et L. Thévenot (1991) offre un moyen d'analyser comment la légitimité des usages est débattue selon différentes logiques. Chaque logique met en avant une conception de l'eau et du monde et un système de légitimité propre. Chaque personne, quel que soit l'intérêt qu'elle défend et l'usage qu'elle veut promouvoir, mobilise peu ou prou ses différentes logiques pour définir un ordre juste, justifiant ses actions et opinions et mettant à l'épreuve celles des autres. Le processus de légitimation collective d'une "nouvelle " gestion de l'eau et de ses usages nécessite une confrontation de ces ordonnancements.

La légitimité des participants est questionnée autour de quatre enjeux : i) quelles qualifications du maître d'ouvrage dans sa capacité à porter l'intérêt général et à piloter le processus; ii) quelle justification de la délimitation de la population concernée, qui comprend le territoire de l'action et les intérêts pris en jeu; iii) quelles qualifications des personnes appelées à participer, c'est-à-dire au nom de quoi sont elles présentes, quelles compétences apportent-elles et iv) quels pouvoirs sont donnés aux participants. Par ailleurs, le pouvoir donné aux usagers dans la décision collective est mis en question étant donné leur intéressement et pose la question de leur place dans le dispositif de gestion. L'article propose une illustration de ces mises à l'épreuve sur le cas du bassin de la Lentilla, dans les Pyrénées Orientales.

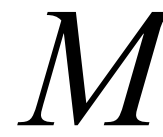

ultiple societal needs and objectives have long been at the root of the debate over arrangements for legitimate uses of water. Furthermore, as these needs and uses evolve, those who feel affected or injured by these changes have become more reserved about these arrangements. The current conflicts therefore reflect a test for old regulatory arrangements of water uses, which lead to two types of questions: is was what was defined as legitimate and just still considered to be so? Are the people and entities involved in the development and operation of these arrangements legitimate to do so?

The "justification" approach suggested by L. Boltanski and L. Thévenot (1991) offers a means of analysing how the legitimacy of uses can be discussed according to various logics. Each logic proposes a conception of water, the world and its own system of legitimacy. Each person, regardless of the interest he or she defends and the use which he or she wants to promote, mobilises to a greater or weaker extent these various logics to define a just order, justifying his or her actions and opinions and putting those of the others to the test. The process of collective legitimation of a "new" water management arrangement of uses requires a confrontation of these elements.

The participants'legitimacy is questioned according to four issues: I) which qualifications does the process manager hold that will allow him or her to represent the general interest and to lead the process; II) What justifications are given for the delimitation around the population concerned, which includes the territory of actions and the interests taken into account; III) What qualifications are required of the people called to take part, i.e. in the name of what are they present, and which competences do they bring; and iv) What powers are given to the participants. The article proposes an illustration of these tests in the case of the basin of Lentilla, in the Pyrenees Orientales. 


\section{I — INTRODUCTION}

L'eau fait partie du quotidien de tout un chacun pour ses besoins domestiques et, pour beaucoup, c'est un élément clé du cadre de vie. Ressources pour de nombreuses activités économiques, l'eau est également un support essentiel d'écosystèmes aquatiques remarquables. Les attentes multiples de la société vis-à-vis de ce bien ont donc suscité de longue date des dispositifs visant à préciser ce qui était jugé légitime de faire avec l'eau. Mais ces besoins et les usages évoluent, induisant des modifications de l'accès à l'eau, de sa disponibilité, de ses qualités ou plus généralement du fonctionnement des écosystèmes aquatiques. Ces dynamiques se heurtent à des réticences de ceux qui se sentent affectés ou lésés. Les conflits reflètent alors une mise à l'épreuve des dispositifs anciens de régulation des usages, avec deux types de questions abordées dans les argumentaires: ce qui était défini comme légitime et juste de faire avec l'eau l'est il toujours? Les personnes et entités impliquées dans l'élaboration et le fonctionnement de ces dispositifs sont-elles légitimes à le faire ?

Ce texte s'appuiera sur différents cas dont celui de la Lentilla ${ }^{1}[1]$, dans les Pyrénées Orientales intéressant en tant qu'il s'ancre dans une longue histoire de gestion locale et de subsidiarité. La première partie de l'article présentera la mise à l'épreuve de la hiérarchie des usages pour définir le bien commun, la seconde traitera des enjeux de légitimation des personnes impliquées dans ce processus.

\section{II $\square$ LÉGITIMATION DES USAGE DE LEAU ET HIÉRARCHISATION POUR UN BIEN COMMUN}

\section{II.1 DES USAGES DANS DES RAPPORTS DE CONCURRENCE DYNAMIQUES : EXEMPLE DE LA LENTILLA}

Le bassin de la Lentilla couvre 9000 ha, sur les Piémonts du Canigou, débouchant dans la retenue de Vinça à sa confluence avec la Têt.

Irrigation et moulins se sont d'abord disputés l'eau et dès le XIII ${ }^{\mathrm{e}}$ siècle, le roi de Majorque octroya un droit d'eau au canal majeur d'irrigation. Puis la prééminence de l'irrigation s'est imposée, les règles d'accès à l'eau agricole, plusieurs fois modifiées, ont régulé le fonctionnement de 7 groupements de propriétaires fonciers, des Associations Syndicales Autorisées d'Irrigation (ASA) qui se partagent un droit de prélèvement de 7001/s pour arroser 650 ha [1]. Ce droit a été accordé en intégrant les usages domestiques par l'alimentation continue des fontaines villageoises avant l'avènement des réseaux AEP. Les usages récréatifs (pêche, baignade), pratiques ancestrales de la population locale rurale, étaient considérés par elles comme secondaires.

1. Ce cas a été étudié dans le cadre d'une thèse en sociologie à paraître Richard-Ferroudji A. L'appropriation des dispositifs de gestion locale et participative de l'eau - Accueillir et composer une pluralité de valeurs, d'objectifs et d'attachements.
Puis l'eau potable de la plaine de Vinça a été captée dans la nappe d'accompagnement de la Lentilla à l'aval de la prise d'eau du canal. La prééminence de l'irrigation a abandonné sa place au profit de l'eau potable. Mais à la fin des années 1960, élus et directeurs d'ASA se sont opposés sur les assecs subits par le captage d'eau potable à l'aval de la prise du canal. Le droit d'eau d'irrigation était plus souvent utilisé à son maximum pour satisfaire une demande agricole plus forte avec l'expansion des vergers. Une solution partielle fut trouvée dans la construction d'un réseau sous pression modernisé avec l'appui de la DDAF, qui supplanta le réseau gravitaire sur $80 \%$ du périmètre. Les besoins des autres usages n'ont pas été négligés, mais ils ne remettaient pas en cause cette hiérarchisation implicite. Par exemple le canal majeur de la plaine alimente la retenue touristique des Escoumes a l'aval, sans pour autant qu'il existe un droit ou une convention. Autre exemple, une ASA qui n'arrosait plus que des jardins de particuliers a été subrogée par une commune afin de maintenir ce service [2].

Les dynamiques récentes bousculent cette hiérarchie des usages. La périurbanisation affecte la plaine de Vinca, ce qui accroît la demande en eau potable, mais amène aussi une population étrangère à la culture locale, qui revendique de l'eau pour des usages domestiques (jardins, piscines) même au sein des ASA puisqu'ils sont aussi propriétaires fonciers. L'emprise de l'agriculture sur le développement local s'effrite. Le Llech, affluent de la Lentilla est devenu un site de canyonning de réputation internationale. La pêche sur les 2 cours d'eau ne concerne plus les seuls résidents. L'accueil d'une population estivale toujours plus nombreuse, attirée par les plans d'eau à l'aval, met à mal la qualité de l'eau des cours d'eau au sortir des stations d'épurations vétustes.

Confrontés à des étiages sévères ( 2 années sur 5 en moyenne), des tensions entre usages ressurgissent à la fin des années 1990, car la dérivation des eaux par les canaux d'irrigation ne laissent plus le débit nécessaire à la préservation du milieu aquatique. Les agriculteurs s'appuient sur leur droit d'eau ancien pour légitimer leur prise d'eau. Les pêcheurs en appellent à la loi sur l'eau de 1984 pour légitimer la prise en compte du milieu aquatique dans la gestion de la Lentilla. La hiérarchie traditionnelle entre les deux usages, qui ne faisait pas tension autrefois puisque les pêcheurs étaient aussi agriculteurs, est mise à l'épreuve. Même si ce qui fait sens pour beaucoup de personnes interrogées c'est la priorité à l'eau potable, la production agricole en second avant les usages de loisirs : baignade, canyoning, pêche ou promenades en pédalo sur les retenues. Ce sens commun partagé par le plus grand nombre explique en partie pourquoi les pêcheurs utilisent de façon précautionneuse le recours à la loi. En effet, pour le Président de la fédération de pêche: «Dans ce monde personne ne comprendrait que les gens défendent âprement une activité de loisir contre les gens qui ont soucis pour en vivre. ». Les pêcheurs préfèrent élaborer un compromis avec les agriculteurs par le biais d'un projet de pompage dans une retenue à l'aval pour réalimenter la Lentilla en période d'étiage sévère. 


\section{II.2 UNE VARIETÉ DE REGISTRE DE LÉGITIMITÉ ET LA QUÊTE D'UN COMPROMIS OU D'UN ARBITRAGE}

Les propos de ce Président révèlent que la perception de la légitimité ne se cantonne pas à une lecture juridique des droits respectifs des parties prenantes. Il ne s'agit pas non plus d'une simple opposition d'intérêts pour s'approprier une ressource ou asseoir un pouvoir. Ce pêcheur souligne l'enjeu de justification morale des décisions et d'une construction d'une juste répartition de l'eau, en référence à des logiques différentes. Chaque logique mettant en avant une conception de l'eau et plus largement du monde, est porteuse de son propre système de légitimité

Différents registres de justification d'une bonne gestion de l'eau

- «Compte tenu des coûts comparés des hypothèses étudiées par le bureau d'étude, l'ensemble des acteurs a opté pour la solution d'augmentation de la ressource, qui permettrait d'assurer dans le cours d'eau un débit compatible avec l'ensemble des usages sans discrimination. ».

Extrait du document de présentation du projet d'aménagement Hydraulique du bassin de la Lentilla

- Un élu, 2005: "Nous sommes pour le maintien des canaux existants (...) pour de multiples raisons, d'une part ça sert encore à l'arrosage dans pas mal d'endroits. Ça sert aussi à l'évacuation des eaux pluviales et c'est quand même des techniques de nos anciens qu'il ne faut pas négliger ».

- Un gérant d'une association de sports d'eau vive, 2005 : "Il faut faire attention, le cours d'une rivière c'est le cours d'une rivière. Pour moi le fait de dire on va remonter de l'eau, c'est aller un peu loin. On va trafiquer, on va vraiment bouleverser, on va faire quelque chose qui n'est pas naturel, on va amener de l'eau dans une zone que pour du profit".

L'approche de la justification proposée par L. Boltanski et L. Thévenot [3] offre un moyen d'analyser comment ce qu'il est légitime de faire est débattu par un ensemble d'acteurs et selon différentes logiques. Dans les situations de dispute, de controverse ou de dénonciation apparaît un besoin d'expliciter les torts et de construire les fondements d'un nouvel accord. La justification permet d'opposer une résistance en cas de critique et de donner une légitimité à un accord. Une pluralité de modes de justification existe. Boltanski et Thévenot identifient 6 principes de référence sur la base de travaux classiques en sciences sociales et sans revendiquer une exhaustivité. Les usagers peuvent mobiliser chacun de ces principes pour une justification sans être attachés de façon définitive à aucun.

L'eau peut être ainsi appréhendée comme une " ressource » (Cf. $1^{\text {er }}$ extrait de l'encadré ci-dessus) dont il convient de rationaliser les usages sur des critères d'efficacité technicoéconomique dans une logique industrielle ou dont il s'agit d'optimiser la valorisation économique dans une logique marchande. Ainsi, l'augmentation de la ressource en eau par pompage dans une retenue permettrait d'optimiser sa valorisation. Selon un principe civique, l'eau est vue comme appartenant à tous et il convient d'agir en toute circonstance avec civisme, tant dans les modalités de prises de décision que dans les pratiques qui l'affectent. Selon un principe domestique, l'eau et ses usages sont appréciées à l'aune des traditions, du patrimoine, de ce qui fait sens commun entre les habitants de la localité. Suivant cette logique, les canaux doivent être respectés comme techniques des anciens (Cf. $2^{\text {ème }}$ extrait de l'encadré). Selon un principe de renom, la grandeur repose sur l'opinion des autres, la notoriété publique telle que la renommée d'un site de pêche de $1^{\text {re }}$ catégorie, un site de canyonning. Dans un monde de l'inspiration, les références portent sur la beauté, les émotions et les actions sur l'eau ou la rivière jugées à l'aulne de ses effets sur les paysages, l'harmonie de la nature. Enfin, dans un monde vert [4], la nature doit être respectée au même titre que les humains (Cf. $3^{\text {ème }}$ extrait de l'encadré.

Chaque personne, quel que soit l'intérêt qu'elle défend et l'usage qu'elle veut promouvoir, peut mobiliser ses différentes logiques pour définir un ordre juste, justifiant ses actions et opinions et mettant à l'épreuve celles des autres. Le processus de légitimation collective d'une «nouvelle» gestion de l'eau et de ses usages comprend une confrontation de ces ordonnancements, à deux niveaux : i) ordonnancement des actions et des objets selon un principe donné ii) mise en ordre des principes entre eux. Le processus est aboutit si émerge un principe ou un compromis entre principes qui fera le «bien commun ».

Sur la Lentilla, la gestion de l'eau est mise à l'épreuve par l'enjeu de préservation du milieu aquatique pourtant celle-ci n'est pas subversive pour la communauté de l'eau existante. Le souci de défense de l'environnement est issu d'une relation familière avec cet environnement et non pas de revendications environnementalistes qui demandent une préservation du milieu aquatique « en général». La plupart des personnes impliquées assument une anthropisation du milieu naturel et sont également soucieuses d'un usage économique de l'eau. Le développement économique de la vallée apparaît comme un enjeu important pour les participants. La discussion porte alors sur l'usage touristique de l'eau vis-à-vis de l'usage agricole. Cependant, on observe également un attachement à l'agriculture comme patrimoine au-delà d'une considération comme activité économique.

"Un président d'ASA 2004 : Je vois, le paysage a drôlement changé en 3 ans, il y avait beaucoup d'arbres fruitiers. Ca devient bizarre. (...) Mon père est décédé il y a un an à peu près. Alors, ces champs, on ne sait pas trop ce que l'on va en faire. Moi, j'aimerais bien les cultiver. J'aimerais bien $y$ revoir des arbres dessus. (...) Mais, après nous qu'est-ce qu'il se passera. Moi mes gosses sont en Angleterre. Avant, les familles avaient plus d'enfants, il y en avait au moins un qui restait à la terre. Mais c'est dommage de voir les choses partir comme ça.»

Ce compromis, dont on pressent les prémices dans les discours de nombreux acteurs de l'eau ou de simples résidents interrogés en face à face, tarde pourtant à s'institutionnaliser aujourd'hui. Sur la Lentilla, l'architecture de gestion de l'eau actuelle s'est construite selon différents registres de justification au fil des siècles, avec une mise en discussion des usages de l'eau à plusieurs reprises [1]. Aujourd'hui, si l'évolution des usages de l'eau vient à nouveau mettre à l'épreuve la communauté de l'eau agricole ce n'est pas une simple concurrence sur la ressource - la nécessité de partage existe aussi entre des groupements d'irrigants et leurs dirigeants s'en sont accommodé au cours des siècles. L'épreuve 
tient d'abord à l'incompréhension entre les personnes, voire à l'absence de dialogue. D'une part, la pénurie d'eau n'est plus perçue par de nombreux usagers qui ne sont pas prêts à s'investir dans des changements de comportements. D'autre part, la communauté de l'eau qui s'était constituée autour d'un droit d'usage agricole de l'eau et des élus a exclu des usagers qui ne sont ni membres de ces associations ni proches des élus et qui pour certains se sentent concernés et intéressés pour s'impliquer.

Un président d'ASA, 2004 "Peut-être les problèmes des gens des villages sont moins liés à l'eau. Actuellement, on a ce que j'appelle des citoyens consommateurs, c'est-à-dire que celui qui ouvre le robinet pour l'eau peut-être il ne sait pas que c'est la mairie qui s'en occupe, il ne connaît que la SAUR qui lui envoie la facture. Il est consommateur et quand on a face à nous au ruisseau ces nouveaux, ils résonnent en consommateur. Ils ne raisonnent pas en citoyens en termes de partage."

C'est la forme héritée de cette communauté de l'eau qui ne permet pas de faire entendre et discuter l'ensemble des voix. On observe bien des adaptations de ce dispositif de gestion pour faire face à cette difficulté, comme la prise de compétence eau d'une communauté de commune ou le projet d'une nouvelle institution pour coordonner l'ensemble des usages sur la plaine de Vinça. Cette dernière offrirait un prolongement du dispositif de mise en politique de l'eau et de gestion subsidiaire. Elle donnerait l'opportunité d'une interaction entre l'ensemble les usagers pour se comprendre avant de faire le travail de montée en généralité pour prendre une décision en visée d'un bien commun. Elle reste cependant soumise à de nombreuses questions liées à l'accueil de la pluralité identifiée en termes d'intérêts, de logiques et de rapports en familiarité avec l'environnement mais également de cultures politiques.

\section{III — LÉGITIMATION DE LA PARTICIPATION DES USAGERS À LA GESTION DE L'EAU}

Le corollaire de ce processus de légitimation des usages tient donc à la composition du groupe et à la qualification des personnes et organisations appelées à y participer ou à définir ensuite les règlements qui en découlent. Jean-Eudes Beuret souligne à propos de concertations environnementales que «le premier sujet de controverse est issu de la question suivante : qui doit être reconnu comme légitime pour participer à la concertation (...) les débats les plus âpres ont lieu en amont de la concertation pour savoir qui y participera » [5 p. 153].

Un ensemble de travaux aborde de façon pragmatique ces processus de légitimation des participants [6-8]. Il ne s'agit plus d'une représentativité structurelle mais d'une représentativité construite et mise à l'épreuve, pour prendre en compte le risque d'usurpation de la parole du représenté. Cet enjeu de légitimation est repris par les guides méthodologiques comme le guide «Le SAGE, mode d'emploi $n^{\circ} 2 »^{2}$. Ce souci de légitimation de chaque participant remplace un idéal de composition prédéfinie que l'on n'aurait qu'à appliquer sur un bassin

2. «Le SAGE, mode d'emploi $\mathrm{n}^{\circ} 2$ » édité par l'Agence de l'eau Rhône - Méditerranée - Corse en septembre 2002. versant. Il ne s'agit plus de se fixer comme objectif d'aller chercher les absents ou des catégories prédéfinies de personnes mais de travailler les mécanismes de mise à l'épreuve des légitimités et d'ouverture à de nouvelles légitimités des participants effectifs. Le dispositif est alors questionné sur l'équipement qu'il propose pour évaluer à tous moments, si besoin est, les légitimités des participants selon différentes dimensions. Les conflits de qualification perturbent la construction du commun ou délégitiment au final, aux yeux de certains, les règlements des usages proposés.

\section{III.1 MISE À L'ÉPREUVE DES LÉGITIMITÉS DES PARTICIPANTS ET ÉVOLUTION DU DISPOSITIF SUR LA LENTILLA}

D'abord, quel dispositif de gestion pourrait donner voix à d'autres usagers que les agriculteurs sur cette plaine. Ensuite, certaines questions posées, comme la protection du milieu aquatique de la Lentilla, dépassent le seul territoire de la plaine de Vinça et remettent en cause un dispositif local de la gestion de l'eau centré sur les ASA.

Les services de l'Etat incitent à organiser la gestion de l'eau à l'échelle de l'ensemble du bassin versant de la Lentilla, en incluant la vallée de son affluent le Llech. La communauté de communes de Vinça Canigou, qui couvre l'ensemble du bassin versant du Llech et de la Lentilla, s'affirme en 2005 comme support de cette gestion en prenant la compétence eau. Elle porte une étude du projet à l'échelle $\mathrm{du}$ bassin versant. Cette prise de compétence répond aux impératifs de gestion territoriale et non plus sectorielle de la loi sur l'eau. Ce dispositif correspond à un idéal selon les critères de la politique française de l'eau dans le sens où une autorité territoriale inclut dans ses mandats la question de l'eau et la traite en lien avec d'autres enjeux. Cependant, il est soumis à deux critiques. D'une part, il renonce à construire une communauté spécifique autour de l'eau puisque la communauté de communes préexiste à sa prise de compétence et qu'elle s'est construite autour de l'enjeu des déchets. D'autre part, il conforte une délégation de la gestion aux élus de la part des usagers.

En 2007, en alternative à la délégation de la gestion à la communauté de commune, une autre solution se dessine. Une fusion des cinq ASA présentes sur la plaine de Vinça est prévue pour se porter maître d'ouvrage du projet qui semble faire consensus au niveau local: un pompage dans la retenue de la Vinça en aval, qui, par remontée de l'eau satisferait l'ensemble des usages. La gestion du pompage reviendrait à une nouvelle institution, composée selon une représentativité liée à l'usage de l'eau sur le modèle des confédérations espagnoles [9]. Les communes représenteraient l'usage « eau potable » et « eau de loisir » en référence aux débits prélevés pour l'eau potable et pour la retenue de loisir des Escoumes. Les pêcheurs seraient présents au titre du débit réservé au milieu naturel. Enfin, les ASA seraient présentes en référence à la quantité d'eau qu'ils prélèvent selon leur droit historique. Cette représentation selon une quantité prélevée maintiendrait le pouvoir des agriculteurs dans la gestion de l'eau, plus important utilisateur en termes de débit. On retrouverait la surreprésentation agricole observée par ailleurs dans les CLE [10] due à un fort investisse- 
ment politique des agriculteurs. Par ailleurs, cette institution se situerait à l'échelle de la plaine de Vinça et non du bassin versant du Llech et de la Lentilla. Le fait de conserver un dispositif à l'échelle de la plaine est présenté comme un atout pour la participation des personnes concernées et leur compréhension mutuelle. Cette institution rassemblerait les personnes en conflit localement et ne s'étendrait ni au bassin affluent du Llech, ni à l'amont de la Lentilla et sans représentant des populations de l'aval potentiellement affectées par les prélèvements dans la retenue de la Vinça.

\section{III.2 DIFFÉRENTS ENJEUX DE LÉGITIMATION DANS UN DISPOSITIF DE GESTION DE L'EAU}

Le cas de la Lentilla constitue un exemple de questionnement de la légitimité à différents niveaux :

i) Quelle est la légitimité du maître d'ouvrage du processus ? Quelle est sa capacité à porter l'intérêt commun et à piloter le processus?

ii) Quelle est la légitimité de la délimitation de la population concernée à travers un territoire de l'action publique ;

iii) Quelle est la légitimité des personnes appelées à participer? Au nom de quoi sont-elles présentes? Quelles compétences apportent-elles ? A quel niveau participent-elles ?

iv) Quels pouvoirs sont donnés aux participants, avec quel équilibre des forces entre intérêts en jeu.

La Lentilla est un exemple d'atermoiement sur le premier critère de qualification du maître d'ouvrage et en corolaire, sur le second, avec la définition du territoire concerné. Les hésitations sur la délimitation du territoire de l'eau pertinent et de la légitimité de la structure porteuse sont communes à beaucoup de bassins et ont contribué à la lenteur de l'émergence des SAGE [11].

La Lentilla pose par ailleurs la question de la représentativité des participants et de leurs compétences. Les dispositifs comme la CLE demandent une participation de représentants des usagers à la gestion de l'eau. L'institutionnalisation de la participation des usagers dans la gestion de l'eau date de la loi sur l'eau de 1964 qui a instauré en France des organes de concertation et de décision incluant des usagers : les comités de bassin et les conseils d'administration des agences de l'eau. Ces dispositifs institutionnels posent la question de la légitimité d'une personne à y être invité alors que le nombre de participants est limité. Le représentant des agriculteurs invité dans la CLE est-il bien représentatif de cette catégorie ? Quels usages considérer pour être représentatif de l'ensemble de la population? Le Bourhis souligne la difficulté de répondre à la question «tout le monde est-il bien représenté? » puisque les absents sont justement muets [10]

La catégorisation en termes d'usagers a conduit au développement et à la structuration de l'eau associative qui se positionne en intermédiaire entre représentants publics et usagers reposant pour ces intermédiaires les questions qui étaient posées aux élus. Quelle est la légitimité de ces participants? Clayes Mekdade a analysé sur le terrain camarguais la constitution des associations en corps intermédiaires « indispensables et inévitables », qui opèrent des opérations de traduction mais reconstituent la problématique de la représentation entre citoyens et Etat républicain et constituent ce qu'elle nomme un système "néo-notabiliaire » [12]. La catégorisation des participants comme usagers en s'appuyant sur un modèle démocratique d'inspiration libérale qui institue la place des lobbies trouve alors ses limites et se reposent les enjeux de représentation et de légitimité dont on avait essayé de se débarrasser en proposant un modèle alternatif à la démocratie représentative. Pour certains élus, les associations et les citoyens n'ont pas la légitimité de défendre l'intérêt général qu'ils ont acquise au suffrage universel direct $[13,14]$.

Pour d'autres élus, les usagers ne sont pas légitimes car insuffisamment compétents et mobilisables et les débats de fond ne peuvent avoir lieu qu'entre spécialistes. Cette vision de l'inutile présence des citoyens - profanes dans ces arènes de discussion d'une part et de la légitime primauté des élus dans les instances de gestion d'autre part est parfois partagée par la majorité de la population. C'est ce que nous ont révélé des sondages d'opinions et des groupes de discussions avec des citoyens dans l'Hérault, dans un contexte de faible mobilisation de la population sur des conflits d'usages latents et peu médiatisés à l'époque [15]. Mais nous avons $\mathrm{pu}$ aussi constater une délégation quasi-totale des débats et des décisions à des représentants élus au sein de communautés d'irrigants, pourtant réputées pour leurs capacités de mobilisation de leurs adhérents, y compris lors de réformes institutionnelles profondes, avec des impacts forts sur les agriculteurs (tarification, droit d'accès à l'eau) [16].

\section{III.3 L'AMBIGUÏTÉ DES USAGERS ENTRE CONTRIBUTION À LA DÉFINITION DU BIEN COMMUN ET DÉFENSE D'INTÉRÊTS PERSONNELS OU SECTORIELS}

En étudiant la constitution de CLE, Le Bourhis montre la difficulté de désignation des porte parole liées aux dangers de manipulation et au risque de surreprésentation d'une catégorie d'usagers comme les agriculteurs, "qui, avec un peu d'astuce, peuvent se retrouver dans le collège des élus » [10]. L'argumentation de la légitimité d'un usager peut toujours être soupçonnée de couvrir une stratégie de défense d'intérêts ou d'objectifs personnels. Cette motivation de l'engagement des personnes vient mettre à l'épreuve le pouvoir donné à un participant lié à la légitimité de son usage de l'eau.

Les doutes portent sur la capacité des usagers ou de leurs porte-parole à se détacher d'intérêts particuliers pour aborder l'intérêt général, surtout dans les projets impliquant des aménagements. Ce soupçon est exprimé par le qualificatif de Nimby (Not In My Backyard). Or, l'analyse des phénomènes Nimby révèle qu'ils portent autant la confrontation d'une pluralité de biens communs que la défense d'intérêts particuliers. Dany Trom montre la capacité des associations à se positionner par rapport l'intérêt général en opérant des «montées en généralité » et transformer la singularité des revendications en un bien commun dont l'expression met à l'épreuve la décision qui s'était stabilisée [17]. «Le travail des militants consiste précisément en la mise en forme d'un bien commun universalisable, susceptible de concurrencer l'intérêt général défini par les autorités traditionnellement habilitées à le déterminer » (p.40). Il souligne la nécessité de convertir la «nature spatialisée» pour fabriquer un 
« singulier général». Ces travaux, comme ceux de Jacques Lolive [18] ont permis d'argumenter la distinction entre traitement local et intérêts privés ou sectoriels en montrant que la gestion locale reste soumise à une justification publique. Les associations apparaissent alors dans toute leur capacité, lorsqu'elles contribuent à l'accommodement des tensions entre l'engagement civique et celui qui tient à un ancrage local ou personnel. Elles servent de «dispositifs d'apprentissage du public. [19]

L'argumentation en public est un exercice délicat. Eric Doidy [20] a montré la difficulté de prise de parole des usagers dans les concertations environnementales. Les lieux de concertation impliquant la rencontre avec d'autres acteurs, exigent alors un détachement et une explicitation verbale de ce qui fonde l'attachement d'une personne à son environnement par une montée en généralité. Les savoirs de proximité doivent s'inscrire dans un cadre plus général comme la préservation du milieu aquatique. Il s'agit de « constituer l'environnement en chose publique » [21]. Cependant, les différentes saisies de l'environnement peuvent être source de tension lors de la coordination des acteurs autour d'un même objet localisé. Il existe une dénonciation réciproque entre «ceux qui pratiquent » une portion de rivière comme les pêcheurs et « ceux qui savent» comme les écologistes.

Or la prise en compte des savoirs pratiques ou propres à un bassin versant représentent un enjeu majeur pour une gestion locale de l'eau qui s'adapte aux particularités de chaque cas. Cecilia Claeys Mekdade montre l'importance des attachements locaux dans la mise en politique des questions environnementales [22]. En dépassant l'opposition entre localisme et globalisme, elle montre l'émergence de la figure légitime du «citoyen localisé » tandis que l'Etat se profile comme protecteur de la diversité nationale. Elle montre, sur le cas du système " socio-hydraulique " camarguais, l'importance de la prise en compte des structures affectives des territoires tout en soulignant deux risques : le «particularisme normalisé » et la « tentation xénophobe ».

\section{CONCLUSIONS : LEGITIMITÉ DES USAGES, DES USAGERS ET GESTION SUBSIDIAIRE DE L'EAU}

En arrière plan de nombreux conflits d'usages de l'eau, il y a la nécessité ressentie par une partie de la population de redéfinir ce qu'il est légitime de faire avec l'eau, avec quelle hiérarchie sur les usages. Deux enjeux de légitimation sont alors sous-jacents : Comment justifier et ordonner dans les revendications des besoins exprimés? Qui est légitime pour argumenter au nom de tel usage ou telle cause ou pour une décision publique?

L'approche de la justification fournit une grille d'analyse des argumentaires énoncés dans ces controverses publiques, en les rattachant à des logiques différentes, chacune avec sa propre échelle de qualification des comportements ou des objets. Ces logiques sont transversales aux intérêts et constituent une autre manière d'appréhender les conflits et leur résolution. Sur le cas de la Lentilla par exemple, nous avons illustré comment un compromis se dessine, fondé sur les logiques industrielle et marchande d'efficacité et de valorisation économique de l'eau qui se mêlent à une vision patrimoniale de l'eau. Ailleurs, ce compromis s'établit sur d'autres bases ${ }^{3}$. Mais ces principes ne sont pas les seuls éléments avancés par les participants dans les réunions sur l'eau, et particulièrement quand il s'agit d'usagers ou de leur représentants. De par les nouvelles lois ces derniers doivent être au moins consultés. Quel rôle réserver à l'usager dans la mise en politique de l'eau?

La figure de l'usager qualifie un participant par ce qui le lie à l'objet de gestion : l'eau. Ce lien peut être considéré de trois manières différentes : comme un intéressement, comme un enjeu à prendre en compte dans une gestion en visée d'un intérêt général ou encore comme un lien personnel et intime avec l'objet de gestion. Souvent, la première considération domine et l'usager est considéré comme celui qui participe pour défendre ses intérêts, comme porteur d'un ou plusieurs enjeux, utilisant de manière stratégique les logiques de portée plus générale. Or, l'usager est également légitime à participer à la construction de l'intérêt commun pour d'autres motifs. De par son lien de proximité avec la ressource, la participation des usagers ou de leurs représentants donne l'occasion d'un ajustement de la gestion au contexte local si le dispositif offre une opportunité d'échanges d'argumentation en visée d'un bien commun enraciné dans le territoire considéré.

La participation des usagers est alors pertinente pour gérer l'eau selon un principe de subsidiarité. Pour Bernard Barraqué, «Dans la subsidiarité, on part de la personne, à laquelle on laisse son autonomie tant qu'elle peut l'assumer. $\mathrm{Si}$ elle ne le peut pas, on cherche à responsabiliser le niveau supérieur, la famille. La famille à son tour s'emboîte dans un niveau corporatif/coopératif supérieur, et l'on remonte ainsi progressivement en cas de besoin jusqu'à l'Etat » [23]. La nouvelle institution envisagée sur la Lentilla, permettrait de faire perdurer une mise en politique de l'eau qui respecte les principes de subsidiarité et la nouvelle hiérarchie des usages, tout en intégrant de nouveaux usagers. Elle privilégie un ancrage local à une délégation de la gestion à des élus bien que ces derniers soient légitimes d'un point de vue civique.

Si le principe de subsidiarité appuie la légitimité de la participation des usagers aux décisions, la tendance en France reste pourtant à la délégation de la gestion. On observe une faible mobilisation de la population pour s'engager personnellement dans les instances de gestion décentralisée sur l'eau, même dans des situations de tension. Manque de connaissance sur les enjeux et défaut de perception des dysfonctionnements ou des risques de la part du grand public sont deux causes possibles, d'après nos observations dans le bassin de l'Hérault. Mais cette absence de mobilisation et la tentation de la délégation s'observent aussi quand les enjeux sont forts et bien identifiés par les usagers. Ainsi, sur le bassin de la Lentilla, comme à Prades en aval [24], et jusque dans les zones d'irrigation plus récentes du sud-ouest on observe une désertion des comités syndicaux des ASA qui fonctionnent sur le bénévolat. Dans ces ASA, les réformes institutionnelles sont portées par un nombre très restreints de syndics [16] et les assemblées générales ne réunissent pas

3. Cf. Thèse d'A. Richard-Ferroudji, déjà citée. 
foule. Thierry Ruf constate que les liens sociaux autour de l'eau semblent de plus en plus faibles [24]. Une hypothèse est que l'engagement familier des personnes avec l'eau a évolué. Pour certains, l'eau c'est celle qui sort du robinet, toujours présente dans le quotidien et égale à elle-même. Elle sait se faire oublier. Pour d'autres, l'eau est capricieuse et il convient d'en prendre soin. Des études socio-anthropologiques sur les attachements des personnes à l'eau et à leur environnement devraient éclairer le concernement des personnes attendues pour participer à la gestion de l'eau. Elles expliqueraient les raisons de l'effritement de l'engagement dans l'action collective. Il y a là un enjeu fort pour le devenir des procédures de gestion locale.

Remerciement à Katherine Daniell pour son aide dans la rédaction de cette communication

\section{V — RÉFÉRENCES ET CITATIONS}

[1] Riaux J. Règles de l'Etat - Règles de la communauté : gouvernance locale de l'eau - Anthropologie comparée de deux systèmes d'irrigation anciens en contexte d'intervention publique : vallée des Aït Bou Guemez (Haut Atlas - Maroc), Plaine de Vinça (Pyrennées - France). In Anthropologie sociale, Edition EHESS 2006. $\mathbf{5 6 2}$

[2] Feraud J. (2003) - Aménagement hydraulique du bassin de la Lentilla. In Edition Chambre d'agriculture des Pyrénées Orientales. 7

[3] Boltanski L., Thevenot L. (1991) - De la justification. Les économies de la grandeur. Paris : Gallimard.

[4] Lafaye C., Thevenot L. (1993) - Une justification écologique? Conflits dans l'aménagement de la nature. Revue française de sociologie. 34 : 495-524

[5] BeURET J.-E. (2006) - La conduite de la concertation pour la gestion de l'environnement et le partage des ressources. L'harmattan.

[6] Rui S. (2004) - La démocratie en débat, Les citoyens face à l'action publique. Armand Colin.

[7] LAFAYE C. (2000) - Gouvernance et démocratie: quelles reconfigurations ?. C. A G. P (eds) : La démocratie à l'épreuve de la gouvernance, Edition Ottawa: Les Presses de l'Université d'Ottawa.

[8] Beuret J.-E., Trehet C. (2001) - Pour la gestion concertée de l'espace rural : appuyer des médiations territoriales. Le courrier de l'environnement de l'INRA.

[9] Barraque B. (1995) - Paris : La Découverte. Les politiques de l'eau en Europe.

[10] Le Bourhis J.-P. (1999) — Le renouvellement des outils de politique de l'eau (SAGE et CLE), résultats et pistes de recherche sur la gestion territoriale de l'eau. In MATE (ed) L'eau en représentation, Gestion des milieux aquatiques et représentations sociales, Edition MATE. 58-72

[11] Allain S. (2002) - La planification participative de bassin : Analyse des conditions d'émergence et des modalités d'élaboration des SAGE. In Edition INRA. volume $\mathbf{n}^{\mathbf{0}} \mathbf{1}$

[12] Claeys-Mekdade C. (2000) - Indispensables ou inévitables corps intermédiaires : représentativité versus traductivité. In Anselme $M$ (ed) Du bruit à la parole, La scène politique des cités, Edition Editions de l'Aube. 311-316

[13] Blatrix C. (1999) - Le maire, le commissaire enquêteur et leur «public ». La pratique politique de l'enquête publique. In Blondiaux L, Marcou G, Rangeon F (eds) : La démocratie locale Représentation, participation et espace public, Edition PUF. 161-176

[14] Busson B (2001) - Le mauvais procès de recours des associations : faux arguments et vrais menaces. Revue juridique de l'environnement. $\mathbf{N}^{\circ} \mathbf{1}:$ : 159-7

[15] Loubier S, Rinaudo J-D, Garin P, Boutet A. (2005) - Preparing public participation at the catchment level: Comparison of three methodologies applied to the Herault river basin. Water Science and Technology. 52 : 33-41

[16] Garin P, LOUBier S. (2006) — Trois réformes des institutions, trois conception du juste au sein des communautés d'irrigant. In Séminaire WADEMED «Nouveaux arrangements institutionnels pour une gestion de la demande en eau ", Edition Cahors.

[17] Trom D. (1999) - De la réfutation de l'effet NIMBY considérée comme une pratique militante. Note pour une approche pragmatique de l'activité revendicative. Revue française de Sciences politiques. 1 : 31-50

[18] Lolive J. (1997) — La montée en généralité pour sortir du Nimby. Politix. 39 : 109-131

[19] Thevenot L. (2001) - S'associer pour composer une chose publique. In Actions associatives, solidarités et territoires, Chopart J.N. et alii (eds), Edition Université de Saint Etienne. : 267-274

[20] Doidy E. (2003) - Faire entendre la voix des usagers dans les concertations environnementales. Sociologie pratique. 7 : 49-64

[21] Thevenot L. (2001) - Constituer l'environnement en chose publique Une comparaison franco américaine. In Blais JP, Gilio $C$, Ion J (eds) : Cadre de vie, environnement et dynamiques associatives, Edition Paris : PUCA. 203-219

[22] Claeys-Mekdade C. (2003) - Le lien politique à l'épreuve de l'environnement Expériences camarguaises. Peter Lang.

[23] Barraque B. (1997) - Subsidiarité et politique de l'eau. In Faure A (ed) Territoires et subsidiarité : l'action publique locale à la lumière d'un principe controversé. Edition Paris : L'Harmattan. 165-201

[24] Ruf T. (2000) - Usages et droits d'eau dans les canaux de montagne Sept siècles d'histoire à Prades (Pyrénées-Orientales). Histoire et sociétés rurales. 16 : 11-44 